\title{
Quit smoking, close your mouth, drop the fork and get off the couch
}

\begin{abstract}
Cardiovascular disease (CVD) including systemic hypertension, diffuse atherosclerosis, valvular heart disease, arrhythmias and coagulopathies can lead to myocardial infarction, cerebrovascular accident or stroke as well as significant peripheral arterial abnormalities with potential loss of digits and limbs. These disease states continue to remain a major contributor of high morbidity and mortality globally placing a great economic burden on the health care systems of all countries. Nowadays, most people have at least one risk factor for CVD, such as diabetes mellitus, hypertension, tobacco smoking, obesity, an unhealthy diet, physical inactivity and psychosocial factors, which make these people more prone to CVD in the near future. It is necessary to address these issues during the early years of life. Perhaps the best strategy would to prevent CVD risk factors through lifestyle modification. We discuss here about the three root causes of all the risk factors of CVD and how we can prevent the development of CVD by adapting to a healthy lifestyle.
\end{abstract}

Keywords: cardiovascular disease, smoking, obesity, diabetes mellitus, exercise, prevention
Volume 7 Issue 4 - 2017

\author{
Niel N Shah,' Parin N Shah, ${ }^{2}$ Muhammad \\ U Dogar, ${ }^{3}$ Sameera Ishtiaq, ${ }^{4}$ Alia Ishtiaq, ${ }^{5}$ \\ Timothy JVittorio ${ }^{6}$ \\ 'Smt. NHL Municipal Medical College, India \\ ${ }^{2}$ BJ Medical College, Civil Hospital, India \\ ${ }^{3}$ Crozer Chester Medical Center, USA \\ ${ }^{4}$ Kingsbrook Jewish Medical Center, USA \\ ${ }^{5}$ SUNY Downstate Medical Center, USA \\ ${ }^{6}$ Bronx-Lebanon Hospital Center, USA
}

Correspondence: Timothy JVittorio, Bronx-Lebanon Hospital Center, Department of Medicine/Division of Cardiology, 1650 Grand Concourse, Bronx, NY 10457, USA, Tel 7185185222 , Fax 5165185585,Email tjvittorio@gmail.com

Received: October II, 2017 | Published: November 15, 2017
Abbreviations: CVD, cardiovascular disease; DM, diabetes mellitus; CNS, central nervous system

\section{Introduction}

In the beginning of the $21^{\text {st }}$ century, chronic disease replaced infectious disease as health problems of concern. Chronic disease such as cardiovascular disease (CVD), obesity, diabetes mellitus (DM), organ carcinoma, chronic obstructive pulmonary disease (COPD), arthritis, osteoporosis, inflammatory bowel disease and degenerative disease of the central nervous system (CNS) are among the most common chronic diseases worldwide. As per the WHO fact sheets, CVD remains the number one cause of death globally. In 2015, approximately 17.7 million people around the world died due to CVD, representing $31 \%$ of all global deaths. ${ }^{1}$ CVD does not only contribute to high morbidity and mortality, but also a heavy economic burden to the health care system nationally as well as globally.

The risk factors for CVD are categorized as modifiable and nonmodifiable risk factors. Modifiable risk factors include obesity, high blood pressure, physical inactivity, high blood glucose and lipid levels, tobacco smoking as well as alcohol abuse. Thus, modifiable risk factors are associated with human behavior, such as dietary habits, physical activity and use of tobacco products. Non-modifiable risk factors include age, heredity or genetic factors and type $1 \mathrm{DM}$. CVD can be prevented by addressing the modifiable or behavioral risk factors using population-wide strategies. In this review, we focus on prevention of CVD risk factors by lifestyle modification and look forward to spreading the words "Quit Smoking, Close Your Mouth, Drop the Fork and Get Off the Couch". Here we discuss how this slogan may assist in reducing the occurrence of CVD.

\section{Quit smoking}

Both clinical and epidemiological research suggest the link between cigarette smoking and the development of respiratory disease (chronic bronchitis, bronchogenic carcinoma and emphysema), CVD including acute myocardial infarction (AMI) and cerebrovascular accident (CVA)/stroke and thromboembolic complications. ${ }^{2-10}$ Cigarette smoke (three main biologically active ingredients in cigarette smoke are nicotine, carbon monoxide and oxidant gases) can cause the endothelial cells to become edematous and inflamed, which ultimately leads to narrowing and remodeling of blood vessels. Additionally, smoking induces the formation of plaque in blood vessels and thus atherosclerosis, which reduces the blood vessel diameter. Furthermore, chemicals in cigarette smoke increase thrombus formation which can occlude the blood vessels while passing through the already narrowed blood vessels and eventually lead to significant symptomatic coronary artery disease (CAD) in the form of an AMI. ${ }^{11}$ According to Burns, ${ }^{7}$ smoking increases the risk of developing CAD by twofold. When smoking is combined with any of the other risk factors for CAD, such as elevated serum lipid concentrations, uncontrolled hypertension and $\mathrm{DM}$, the risk of CAD increases to the extent of eightfold.

The INTERHEART study ${ }^{12}$ showed a clear dose-response relationship between smoking and the risk of AMI. The study demonstrated the deleterious effects of smoking even in those who were smoking less cigarettes. Those who smoked 1 to 5 cigarettes per day experienced a $40 \%$ increase in the risk of AMI compared with nonsmokers, while those who smoked 6 to 10 cigarettes per day had a twofold increase in risk, and those who smoked 20 cigarettes per day had a fourfold increase in risk. All smoker subjects had similar results irrespective of the device used for tobacco smoking (filtered or nonfiltered cigarettes, bidis, pipes or cigars). ${ }^{12}$

Furthermore, smoking increases the risk of CVA/stroke and deaths occur more likely in smokers than former smokers or non-smokers. ${ }^{11}$ Cigarette smoking can also increase the risk of peripheral arterial disease (PAD) by making the blood vessels narrower as previously discussed. Thus, smoking can be considered as the most common preventable cause of PAD. ${ }^{11}$ Additionally, smoking is a known cause of aortic aneurysms, in particular those involving the abdominal aorta, which is a life-threatening condition. Smoking is responsible 
for the progression of an already existing aortic aneurysm by inducing expansion or rupture of the aneurysm. Interestingly, female smokers have a higher risk of dying from aortic aneurysm than their male counterpart smokers. In young adults who started smoking in their adolescent years, autopsies have shown early narrowing of their abdominal aorta. ${ }^{13}$

\section{Close your mouth and drop the fork}

Obesity is a pathophysiological condition caused by excessive accumulation of fat in the body. This excess fat can increase morbidity, mortality and health care expenditure, while reducing the quality of lifestyle. Obese people are usually more prone to develop metabolic syndrome, which is characterized by the combination of hypertension, insulin resistance and dyslipidemia. These pathological abnormalities can ultimately place obese persons at the risk of type $2 \mathrm{DM}, \mathrm{CAD}$ and cerebrovascular disease. An unhealthy dietary habit is one of the two vital factors (physical inactivity being second) for the development of obesity and thus indirectly responsible for these comorbidities. In addition to the development of long-term health problems, obesity increases one's work disability, unhealthy life years and duration as well as frequency of hospitalization. It is very well established that obesity can lead to type $2 \mathrm{DM}$ and uncontrolled DM can result in atherosclerosis and eventually CAD. ${ }^{14-18}$ The Framingham study has shown that the incidence of AMI in patients with DM is two times greater in males and three times greater in females compared to a healthy population. ${ }^{19}$

Humans need food that is properly balanced with macronutrients (carbohydrates, proteins and fats) and micronutrients (vitamins and minerals) to meet their body's growth and daily energy requirements. As we previously discussed, unhealthy dietary habits, obesity and type $2 \mathrm{DM}$ are associated with one another and type $2 \mathrm{DM}$ is consistently linked with a high risk of CVD. Therefore, by adapting healthy dietary habits, a person can prevent the development of CVD. ${ }^{20}$ More specifically, the following dietary adaptations can be made to prevent CVD especially in the type $2 \mathrm{DM}$ patients. ${ }^{21}$ Reduction in the daily calorie intake (by 500 to $800 \mathrm{kcal} /$ day), food portion sizes and total fat intake (mainly saturated fat); increase the amount of dietary fibers in daily meals and moderate alcohol intake.

There is great evidence that diets rich in vegetables, fruits, high fiber cereals, whole grains, low-fat dairy, fish and diets low in sodium and saturated fat, can markedly reduce the risk of obesity and CVD. ${ }^{22}$ Additionally, it is important to maintain a diet which consists of foods with a low glycemic index. The low glycemic load seems to result in homeostasis of the satiety and hunger hormones (leptin and gherlin). As a result, glucose levels remain lower and the characteristic rise in insulin is blunted. This is a safe way to obtain both weight loss and maintenance. ${ }^{23}$

The effects of dietary fat intake and prevention of CVD was assessed by Hooper et al., ${ }^{24}$ in a systematic review of 27 studies. They included data from randomized placebo-controlled clinical trials with duration of at least six months to two years in their metaanalysis and the included trials consisted of the following dietary interventions: change from intake of saturated fat to unsaturated fat or reduced intake of total fat, saturated fat and dietary cholesterol. The results showed the reduction in the cardiovascular (CV) events (AMI, CVA, etc.) by $16 \%$ and CV mortality by $9 \%$ in subjects who were following the appropriate dietary interventions such as reduction or modification of dietary fat intake. Data from trials with a two-year follow-up period provided better evidence of protection from $\mathrm{CV}$ morbidity and mortality after the reduction or modification of dietary fat or cholesterol intake, when compared with the trials from the sixmonth follow-up period. Furthermore, the dietary fat trials lasting over two years showed up to a $24 \%$ reduction in CV events. Thus, dietary interventions such as continuous reduced intake of dietary fat and cholesterol or modification in dietary fat intake, can play an important role in the prevention of CVD. ${ }^{25}$

Meanwhile, dietary fibers are known to play a vital role in $\mathrm{CV}$ health and gastrointestinal function. ${ }^{11}$ Dietary fibers are of two types, soluble and insoluble. Soluble fibers help to lower the blood cholesterol levels (especially low-density lipoprotein cholesterol or LDL-C) and the rate of glucose absorption from the intestine which helps in preventing spikes in blood sugar concentrations especially in DM patients. ${ }^{11}$ On the other hand, insoluble fibers are responsible for softening the stools, increasing peristalsis of gastrointestinal tract and trapping a wide variety of materials, such as bile salts and minerals. This trapping of bile salts helps to improve CV health by reducing the absorption of cholesterol. ${ }^{11}$

\section{Get off the couch}

Physical inactivity is another vital factor for development of obesity and associated co-morbidities. More than 250,000 yearly deaths in the United States are attributed to CVD resulting from a lack of physical activity. On the other hand, physical inactivity is estimated to cause $30 \%$ of CAD. ${ }^{26}$ As the association between physical inactivity and the increased risk of CV morbidity solidified, further data and studies support the advantages of exercise on physical wellbeing. The inherent advantages of physical exercise stem from an increase in the cardiac output and an enhancement of the innate ability of muscles to extract and to utilize oxygen from the blood. ${ }^{27}$ This benefit is further compounded by the benefit physical exercise has on high- density lipoprotein cholesterol (HDLC), ${ }^{28}$ adipose tissue distribution, ${ }^{29}$ increased insulin sensitivity, ${ }^{30}$ improved cognitive function, ${ }^{31}$ enhanced response to psychosocial stressors, ${ }^{32}$ as well as determent of depression. ${ }^{33}$ With the benefit of physical exercise well established, the question remains which type of exercise provides the most effective and efficient means to help deter CVD.

Aerobic exercise is defined as any activity that uses large muscle groups, can be maintained continuously and is rhythmic in nature. ${ }^{34}$ As the name implies, muscle groups activated by this type of exercise rely on aerobic metabolism to extract energy in the form of adenosine triphosphate (ATP) from amino acids, carbohydrates and fatty acids. Examples of aerobic exercise include cycling, dancing, hiking, jogging/long distance running, swimming and walking. Various studies have been published that prove the advantages of aerobic exercise in reversing and preventing CVD. In 2002, Wisløff et al., ${ }^{35}$ were the first to show the benefit of aerobic training in the myocardium after an ischemic event. Their study was performed on adult female SpragueDawley rats, which were placed into groups categorized based on induced myocardial infarction (MI) with and without exercise and controls with and without exercise. The results showed a $15 \%$ reduction in the left ventricle (LV) hypertrophy postinfarction, as well as $12 \%$ and $20 \%$ decreases in myocyte length and width, respectively, with aerobic exercise. Furthermore, a $60 \%$ improvement was noted in myocardial contractility in subjects with a MI who were assigned to the training group, suggesting enhanced myocardial $\mathrm{Ca}^{2+}$ sensitivity. They were able to conclude the beneficial effects of aerobic training on cardiac remodeling and myocardial contractility. ${ }^{35}$ 
Additionally, aerobic exercise has shown to have a positive impact on other dimensions of cardiovascular health. Several studies have shown that aerobic exercise improves the lipid profile, particularly increasing the HDLC. ${ }^{36}$ In an Australian study, aerobic exercise led to a small but statistically significant reduction in total cholesterol (TC), low-density lipoprotein cholesterol (LDLC) and triglycerides (TG) ranging in a span of $0.08 \mathrm{mmol} / \mathrm{L}$ to $0.10 \mathrm{mmol} / \mathrm{L}$. They also showed an increase in HDLC with their aerobic exercise program of about $0.05 \mathrm{mmol} / \mathrm{L}$. While aerobic exercise appears to have some beneficial effects, its contribution is limited on frequency and quantity. A very recent publication by a Danish group was able to represent what they called a "U shaped association" between aerobic exercise and mortality. Their research quantified 1 to $2.4 \mathrm{~h}$ of exercise over 2 to 3 times per week as the optimal quantity and frequency standard of aerobic exercise to promote improved health. Interestingly, they quantified any amount above that standard as being indifferent to the mortality risk, as that of sedentary individuals. ${ }^{37}$

On the other hand, anaerobic exercise is defined as intense physical activity of very short duration, fueled by the energy sources within the contracting muscles and independent of the use of inhaled oxygen as an energy source. ${ }^{38}$ Without the use of oxygen, our cells revert to the formation of ATP via glycolysis and fermentation. This process produces significantly less ATP than its aerobic counterpart and leads to the build-up of lactic acid. Examples of anaerobic exercise include sprinting, high-intensity interval training (HIIT), power-lifting, etc. Similar to aerobic exercise and their favorable effect on lipid metabolism, anaerobic exercises have been shown to have a positive influence on the lipid profile. A small European study composed of 16 obese subjects was able to show the increased benefits of an aerobic workout followed by anaerobic training, as compared to aerobic training alone. Subjects who underwent core training with both aerobic and anaerobic exercises showed a larger reduction in non-esterified fatty acids. The same group was also found to have the greatest reduction in their body mass index (BMI). ${ }^{39}$

In a Turkish study completed by Akseki Temür et al., ${ }^{40}$ the effects of anaerobic exercise were evaluated with a member of the natriuretic peptide family, known as C-type natriuretic peptide (CNP). CNP is synthesized by the endothelium and offers a protective effect through its effects on the vascular tone of blood vessels, well as exerting anti-fibrotic and anti-proliferative properties. It produces a hyperpolarization effect on the smooth muscle layer of blood vessels, which causes vasodilatation. ${ }^{41} \mathrm{CNP}$ has also been reported to exert its non-proliferative effects on cardiac fibroblasts to help prevent cardiac aging through LV fibrosis via the cyclic guanosine monophosphate (cGMP) pathway..$^{42}$ Both aerobic and anaerobic exercises have unique and collective positive correlations towards improved cardiovascular health. Despite all the research, further studies are still warranted to delve further into the impact that both aerobic and anaerobic exercise may have on human physiology to unequivocally determine if there is superiority of one type of exercise over another. ${ }^{27}$

\section{Conclusion}

As the prevalence of CVDs and the people with CVD risk factors are increasing, we need to focus on the root causes for the CVD risk factors and address those by making a good preventive strategy. The three major root causes responsible for all CVD risk factors are tobacco smoking, unhealthy dietary habits and physical inactivity. It is necessary to adopt a good integrative preventive approach that include lifestyle modifications, such as smoking cessation, healthy dietary habits and regular physical exercise. By implementing such preventive approach in our life, we would be able to reduce the cardiovascular morbidity, mortality, hospitalization rate and also the health care costs. Therefore, it would be advisable to follow the words "Quit Smoking, Close Your Mouth, Drop the Fork and Get off the Couch" to live a healthy and better life.

\section{Acknowledgements}

None.

\section{Conflicts of interest}

The author declares no conflict of interest.

\section{References}

I. World Health Organization. Cardiovascular Diseases (CVDs). 2017.

2. Janzon E, Hedblad B, Berglund G, et al. Changes in blood pressure and body weight following smoking cessation in women.J Intern Med. 2004;255:266-272

3. Paulus D, Saint-Remy A, Jeanjean M. Smoking during adolescence: Association with other cardiovascular risk factors in Belgian adolescents. Eur J Public Health. 2000; 10:39-44.

4. Polidori MC, Mecocci P, Stahl W, et al. Cigarette smoking cessation increases plasma levels of several antioxidant micronutrients and improves resistance towards oxidative challenge. Br J Nutr. 2003;9(I): I 47-I50.

5. Wiggers LC, Smets EM, de Haes JC, et al. Smoking cessation interventions in cardiovascular patients. Eur J Vasc Endovasc Surg. 2003;26(5):467-475.

6. Benowitz NL. Cigarette smoking and cardiovascular disease: Pathophysiology and implications for treatment. Prog Cardiovasc Dis. 2003;46(I):9I-III.

7. Burns DM. Epidemiology of smoking-induced cardiovascular disease. Prog Cardiovasc Dis. 2003;46(I): I I-29.

8. Taylor DH Jr, Hasselblad V, Henley SJ, et al. Benefits of smoking cessation for longevity. Am J Public Health. 2002;92(6):990-996.

9. Edwards R. The problem of tobacco smoking. BM]. 2004;328(7433):217219

10. Goldenberg I, Jonas M, Tenenbaum A, et al. Current smoking, smoking cessation, and the risk of sudden cardiac death in patients with coronary artery disease. Arch Intern Med. 2003;163:230I-2305.

II. Buttar HS, Li T, Ravi N. Prevention of cardiovascular diseases: Role of exercise, dietary interventions, obesity and smoking cessation. Exp Clin Cardiol. 2005; I0(4):229-249.

12. Yusuf S, Hawken S, Ounpuu S, et al. Effect of potentially modifiable risk factors associated with myocardial infarction in 52 countries (the INTERHEART study):case-control study. Lancet. 2004;364(9438):937952

13. Norman PE, Curci JA. Understanding the effects of tobacco smoke on the pathogenesis of aortic aneurysm. Arterioscler Thromb Vasc Biol. 2013;33(7): 1473-1477.

14. Visscher TL, Rissanen A, Seidell JC, et al. Obesity and unhealthy life-years in adult Finns: An empirical approach. Arch Intern Med. 2004;164:14131420.

15. Clinical guidelines on the identification, evaluation, and treatment of overweight and obesity in adults: Executive summary. Expert Panel on the Identification, Evaluation, and Treatment of Overweight in Adults. Am J Clin Nutr. 1998;68(4):899-9|7.

16. World Health Organization. Obesity and overweight. 2005. 
17. Grundy SM. Obesity, metabolic syndrome, and cardiovascular disease.J Clin Endocrinol Metab. 2004;89(6):2595-2600.

18. Pi-Sunyer FX.The epidemiology of central fat distribution in relation to disease. Nutr Rev. 2004;62: I20S-126S.

19. Kannel WB, McGee DL. Diabetes and cardiovascular risk factors: The Framingham study. Circulation. 1979;59(1):8-13.

20. de Lorgeril M, Salen P. Diet and the prevention of coronary heart disease. In: Arnoldi A, editor. Functional Foods, Cardiovascular Disease and Diabetes. USA: CRC Press; 2004. p. 2 I-48.

21. Khazrai YM, Manfrini S, Pozzilli P. Diet and diabetes: Prevention and control. In: Arnoldi A, editor. Functional Foods, Cardiovascular Disease and Diabetes. USA: CRC Press; 2004. p. I26-14I.

22. de Roos NM. The potential and limits of functional foods in preventing cardiovascular disease. In:Arnoldi A, editor. Functional Foods, Cardiovascular Disease and Diabetes. USA: CRC Press; 2004. p. I-6.

23. Shah NN, Dogar MU,Vittorio TJ.The Role of Neurohormonal Imbalances in Obesity. Adv Obes Weight Manag Control. 2017;7(I):00182.

24. Hooper L, Summerbell CD, Higgins JP. Dietary fat intake and prevention of cardiovascular disease: Systematic review. BMJ. 200I;322(789):757763.

25. Gunness P, Gidley MJ. Mechanisms underlying the cholesterollowering properties of soluble dietary fibre polysaccharides. Food Funct. 2010;I(2): I49-155.

26. Myers J. Cardiology patient pages. Exercise and cardiovascular health. Circulation. 2003; 107:2e-5e.

27. Patel H, Alkhawam $H$, Madanieh $R$, et al. Aerobic vs anaerobic exercise training effects on the cardiovascular system. World J Cardiol. 2017;9(2):134-138.

28. Williams PT. High-density lipoprotein cholesterol and other risk factors for coronary heart disease in female runners. $N$ Engl J Med. 1996;334:1298-1303.

29. Schwartz RS, Shuman WP, Larson V, et al. The effect of intensive endurance exercise training on body fat distribution in young and older men. Metabolism. I99|;40(5):545-55।.

30. Rosenthal M, Haskell WL, Solomon R, et al. Demonstration of a relationship between level of physical training and insulin-stimulated glucose utilization in normal humans. Diabetes. 1983;32(5):408-4I I.
31. Spirduso WW. Physical fitness, aging, and psychomotor speed: a review.J Gerontol. 1 980;35(6):850-865.

32. Crews DJ, Landers DM. A meta-analytic review of aerobic fitness and reactivity to psychosocial stressors. Med Sci Sports Exerc. 1987;19(5 Supp): I I4S-I 20S.

33. Lobstein DD, Mosbacher BJ, Ismail AH. Depression as a powerful discriminator between physically active and sedentary middle-aged men. J Psychosom Res. 1983;27(I):69-76.

34. Wahid A, Manek N, Nichols M, et al. Quantifying the Association Between Physical Activity and Cardiovascular Disease and Diabetes:A Systematic Review and Meta-Analysis. J Am Heart Assoc. 20 16;5(9):e002495.

35. Wisløff U, Loennechen JP, Currie S, et al. Aerobic exercise reduces cardiomyocyte hypertrophy and increases contractility, $\mathrm{Ca}^{2+}$ sensitivity and SERCA-2 in rat after myocardial infarction. Cardiovasc Res. 2002;54(I): I62-I74.

36. Blumenthal JA, Emery CF, Madden DJ, et al. Effects of exercise training on cardiorespiratory function in men and women older than 60years of age. Am J Cardiol. 1991;67(7):633-639.

37. Schnohr P, O'Keefe JH, Marott JL, et al. Dose of jogging and longterm mortality: the Copenhagen City Heart Study. J Am Coll Cardiol. 20I5;65(5):4II-4I9.

38. American College of Sports Medicine. ACSM's guidelines for exercise testing and prescription. USA: Lippincott Williams \& Wilkins; $20 \mathrm{I} 3$.

39. Salvadori A, Fanari P, Marzullo P, et al. Short bouts of anaerobic exercise increase non-esterified fatty acids release in obesity. Eur J Nutr. 20I4;53(I):243-249.

40. Akseki Temür H,Vardar SA, Demir M, et al.The alteration of NTproCNP plasma levels following anaerobic exercise in physically active young men. Anatol J Cardiol. 2015; 15:97-102.

4I. Chauhan SD, Nilsson H,Ahluwalia A, et al. Release of C-type natriuretic peptide accounts for the biological activity of endothelium- derived hyperpolarizing factor. Proc Natl Acad Sci USA. 2003; | 00(3):|426-| 43 I.

42. Sangaralingham SJ, Huntley BK, Martin FL, et al. The aging heart, myocardial fibrosis, and its relationship to circulating $\mathrm{C}$-type natriuretic Peptide. Hypertension. 20 I 1;57:201-207. 